POTENTIAL OF THE ACOUSTO-ULTRASOUND METHOD TO CHARACTERIZE THE STRENGTH OF HOT GAS FILTER MATERIALS

\author{
P. Pastila, A.-P. Nikkilä and T. Mäntylä \\ Tampere University of Technology \\ Institute of Materials Science \\ P.O. Box 589 \\ 33101 Tampere, Finland
}

W. Ellingson, E. R. Koehl and D. Sandberg

Argonne National Laboratory

Energy Technology Division

9700 South Cass Ave., Bldg. 212

Argonne, IL USA 60439-4838

December 2001

The submitted manuscript has been created by the
University of Chicago as Operator of Argonne
National Laboratory (ÒArgonneÓ) under Contract No.
W-31-109-ENG-38 with the U.S. Department of
Energy. The U.S. Government retains for itself, and
others acting on its behalf, a paid-up, nonexclusive,
irrevocable worldwide license in said article to
reproduce, prepare derivative works, distribute copies
to the public, and perform publicly and display
publicly, by or on behalf of the Government.

Abstract submitted for presentation at the American Ceramic Society's 26th Annual International Conference on Advanced Ceramics \& Composites, January 13-18, 2002, Cocoa Beach, Florida.

${ }^{t}$ Work supported by the U.S. Department of Energy, Office of Fossil Energy/Advanced Research and Technology Development Materials Program, and Clean Coal Technology/Hot-Gas Cleanup Program, under Contract W-31-109-Eng-38. 


\title{
POTENTIAL OF THE ACOUSTO-ULTRASOUND METHOD TO CHARACTERIZE THE STRENGTH OF HOT GAS FILTER MATERIALS
}

\author{
P. Pastila, A.-P. Nikkilä, and T. Mäntylä \\ Tampere University of Technology \\ Institute of Materials Science \\ P.O. Box 589 \\ 33101 Tampere, Finland \\ W. Ellingson, E. R. Koehl, and D. Sandberg \\ Argonne National Laboratory \\ Energy Technology Division \\ 9700 South Cass Ave., Bldg. 212 \\ Argonne, IL USA 60439-4838
}

\begin{abstract}
The objective of this work was to assess the potential of acousto-ultrasonic nondestructive evaluation for characterizing the strength of hot gas filter materials based on clay-bonded SiC and alumina/mullite. Samples were exposed to high-temperature in air and air/water vapor environments, the stress wave factor (SWF) was determined by the acousto-ultrasound method, and the hoop strength was obtained by internal burst testing. The samples were further analyzed microstructurally using electron microscopy and x-ray diffraction. Density and porosity were obtained using the Archimedes method. A correlation of hoop strength with averaged SWF was found. The variation in SWF and strength along the samples is discussed in terms of their relationship to density and porosity.
\end{abstract}

\section{INTRODUCTION}

Hot gas filtration is employed to clean the fuel gas and improve the efficiency of high-temperature, highpressure coal combustion and gasification power conversion processes. Oxide and nonoxide ceramic candle filters have shown the most promise for a decade now because of their durability at high-temperature operation environments. ${ }^{1,2}$ But even these materials have suffered from microstructural changes at both real ${ }^{1}$ and laboratory ${ }^{3}$ operation environments. These changes affect the retained strength of the materials and their reliability.

A nondestructive evaluation method for hot gas filter materials has been under development at Argonne National Laboratory. This acousto-ultrasound (AU) $\operatorname{method}^{4-6}$ is a combination of traditional ultrasonics and acoustic emission measurements; an ultrasonic pulse or stress wave is sent to the material by a transmitting transducer, and another receiving transducer detects the displacements of the material due to the proceeding stress wave. The wavelength of the transmitted pulse is chosen to produce a Lamb wave that occupies the whole volume of material between the transducers ${ }^{6}$. The wave propagation is affected by scattering from microstructural features like porosity, cracks, and differences in the elastic properties of the phases present, spreading of the wave beam, and absorption and splitting of the beam energy due to mode conversions ${ }^{7}$. This effect is determined by changes in the AU parameters such as stress wave factor (SWF), ${ }^{4}$ which in this paper is a simple ring-down count quantifying the AU signal.

According to Aduda and Rawlings, ${ }^{8}$ AU has been applied to monitor the strength of polymer matrix composites, crystallization in glass ceramics, porosity and strength of porous gypsum, and the thermal shock behavior of alumina and zirconia-toughened alumina. Results by Ellingson et al. ${ }^{4-6}$ show that AU can be applied to several types of ceramic hot gas filters, and that the SWF follows the residual strength of the materials. Also, results from Kathrina and Rawlings ${ }^{9}$ indicate a correlation between the strength of sintered $\mathrm{MgO}$ and ring-down count, but this has a negative slope, whereas the correlation for hot gas filters has a positive slope.

Recognizing the possible effect that microstructural features may have on AU results, this work is an attempt to assess the microstructural features that account for variations in SWF. For this effort, two types of filter material were exposed to conditions that cause microstructural and strength changes. The SWF data were collected from several locations along the samples and associated with appropriate ring specimens for hoop 
strength. There was significant variation in SWF along the samples, and some of that variation could be explained by variation in the porosity of the filters.

\section{MATERIALS AND METHODS}

The samples were taken from hot gas filters composed of commercial-grade SiC ("S") and alumina/mullite ("Q"). One sample of each type was exposed in air at $900^{\circ} \mathrm{C}$ for 2000 hours, later denoted as S1 and Q1. Two other samples, S2 and Q2, were exposed in air and water vapor at $900^{\circ} \mathrm{C}$ for 670 hours. Typically, the sample was a 20-30 cm long segment cut from a filter with outer diameter of $60 \mathrm{~mm}$ and inner diameter of $40 \mathrm{~mm}$.

The samples were studied by acousto-ultrasound. At $150 \mathrm{kHz}$ center frequency, the pulsed transducer produces a Lamb wave that travels along the sample wall and is finally detected by the receiving transducer. The SWF is calculated from the received signal as number of amplitudes that exceed a preset threshold. The threshold was different for each material, and the data are thus to be compared only within a single material type. Momentary contact transducers were coupled with spring loading and were thus movable as a pair. The SWF was acquired along the length of the filter at three 93 imuthal locations: $0^{\circ}, 120^{\circ}, 240^{\circ}$. Along each data acquisition path, data were taken from several overlapping positions along the sample. Seven pulses were sent and analyzed for each position. Later, the samples were cut into 20 -mm-wide rings for the strength measurements with an internal hydraulic pressure test ${ }^{10}$.

Figure 1 shows the locations of the acquired SWF data and associated strength. Four strength specimens were associated with one axial SWF location. Note however that at any one axial location, SWF data were obtained at three 93 imuthal positions: $0^{\circ}, 120^{\circ}, 240^{\circ}$. The overall SWF is the average of SWFs from all locations. The local SWF is the average of SWFs from the same axial position on all three axes. The SWF of a position is an average of seven pulses. The porosity data are associated with the positions and locations in a similar way. Open porosity and apparent density of specimens were obtained from several axes. The Archimedes method was used with boiling water. Microstructural characterization included scanning electron microscopy (SEM), energy dispersive spectroscopy (EDS), and X-ray diffraction (XRD).

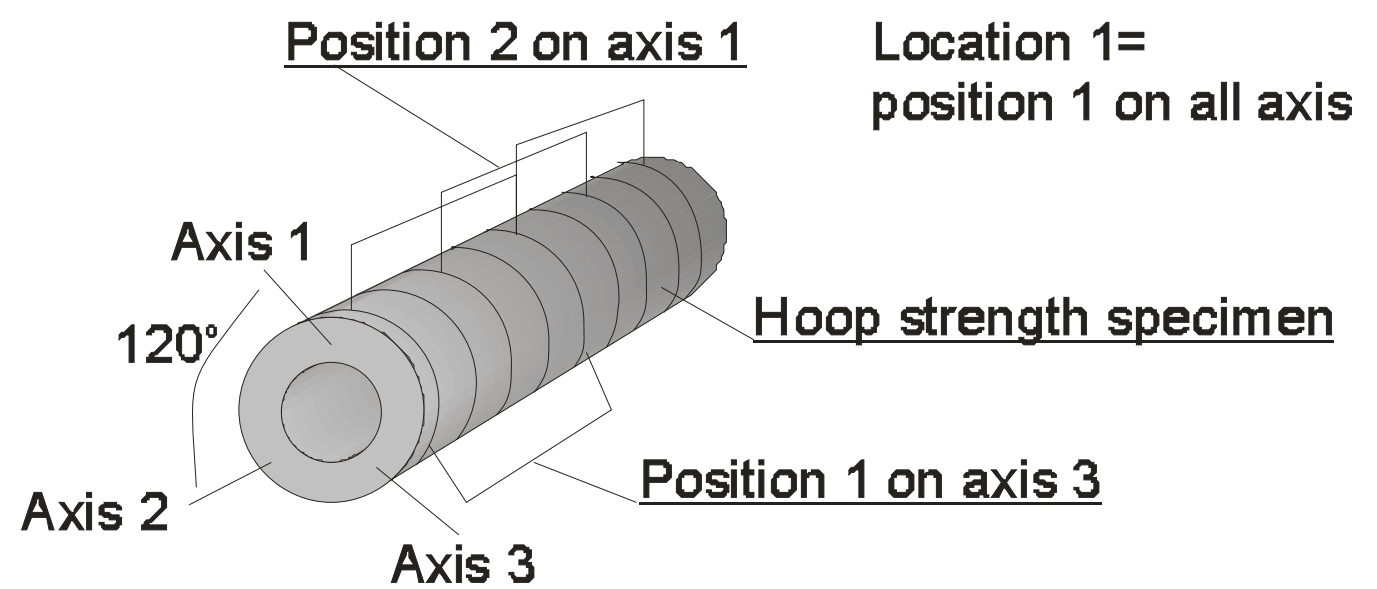

Fig. 1. Positions of acquired SWF and associated strength specimen.

\section{RESULTS}

The modulus of rupture (MOR) strength, its 90\% confidence range [(1-a) $=0.9]$, the SWF, and its standard deviation calculated from the local SWF values are presented in Table I. These values represent the whole filter samples; the MOR is calculated from the data for all strength specimens, and the SWF is an average of all measured SWF values from 9 different positions for S filters and 12 positions for $\mathrm{Q}$ filters. The standard deviation of the local SWF value determined from seven measurements at each position was 1.1 or less. When MOR and SWF are compared, the increase in SWF corresponds to an increase in strength, as demonstrated several times before for hot gas filter materials. In this case, both the differences in SWFs and in strength are very small, and in a strict sense, strength data of samples 1 and 2 for each filter is the same. 
Table I. MOR strength, 90\% confidence range, SWF, and its standard deviation calculated from the local SWF values of the filter samples.

\begin{tabular}{|l|l|l|l|l|}
\hline Filter & MOR $[\mathrm{MPa}]$ & MOR $(1-\mathrm{a}=0.9)$ & SWF & SWF STDEV \\
\hline Q1 & 18.5 & $17.7-19.4$ & 263 & 5 \\
\hline Q2 & 18.1 & $17.6-18.6$ & 257 & 17 \\
\hline S1 & 17.0 & $16.0-18.1$ & 231 & 13 \\
\hline S2 & 17.8 & $16.9-18.6$ & 220 & 24 \\
\hline
\end{tabular}

The microstructural study did not reveal significant differences in the microstructure of filters Q1 and Q2. The XRD spectra suggested that sample Q2 contains slightly more cristobalite than sample Q1. This is possible since water vapor present in exposure of Q2 is known to help crystallization of an amorphous phase. Sample S2 was also expected to contain more cristobalite than S1, but quantitative analysis of XRD data showed no difference. Large variations in porosity of the filters were seen in the SEM investigation, Fig. 2.
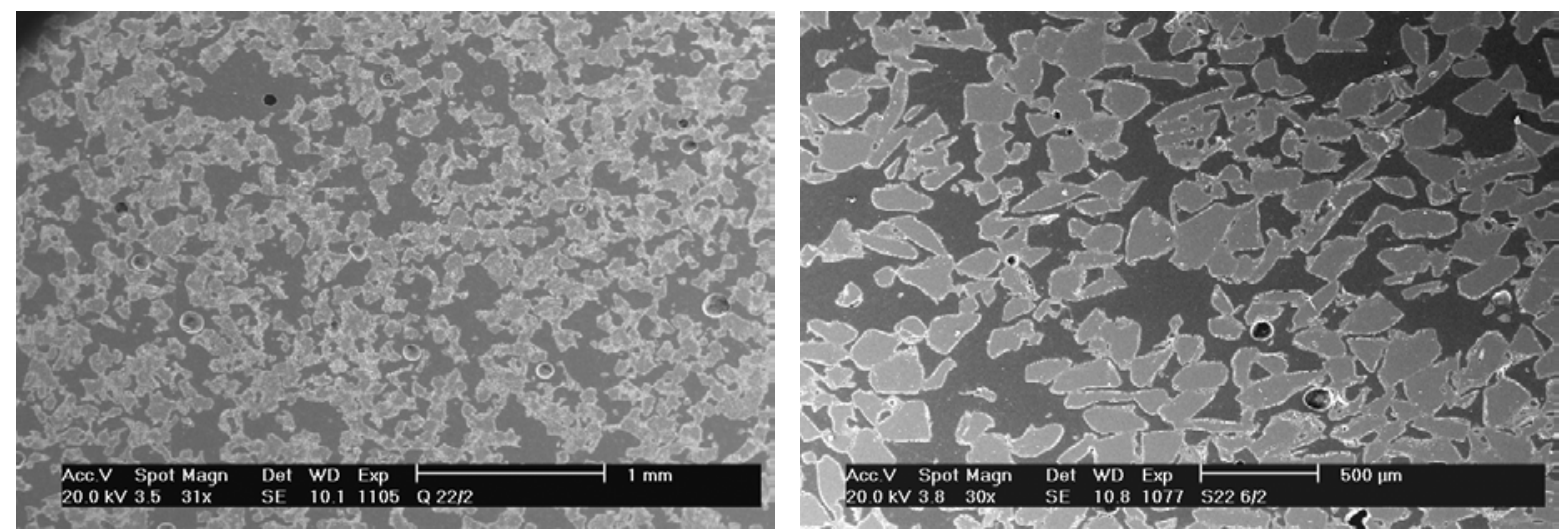

Fig. 2. Variation on the porosity in polished cross sections of filter samples.

On the left, sample Q2, on the right, sample S2.

The local SWF and open porosity along samples are presented in Fig. 3. The local SWF number is an average of seven pulses analyzed from the same position. The porosity is an average of specimens associated with each SWF position as presented in Fig. 1. The standard deviation of the porosity values was at worst $0.3 \%$ but typically around $0.2 \%$ or less. The SWF values of filter Q2 axis 2 and 3 seem to follow the porosity data. Also, the variation of SWF in filter S2 at location 2 follows the variation in porosity. The standard deviation of the apparent density was of same order as changes in it, so it did not give any further information. 


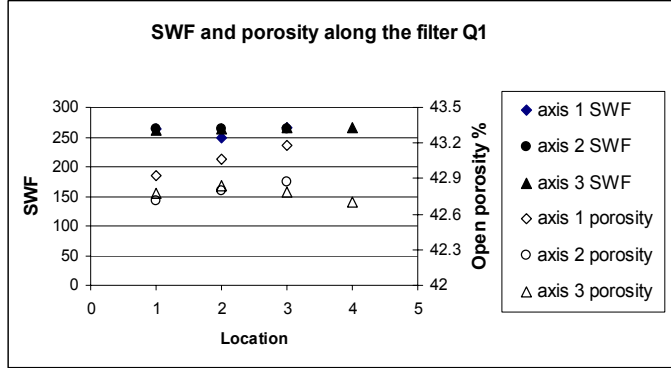

(a)

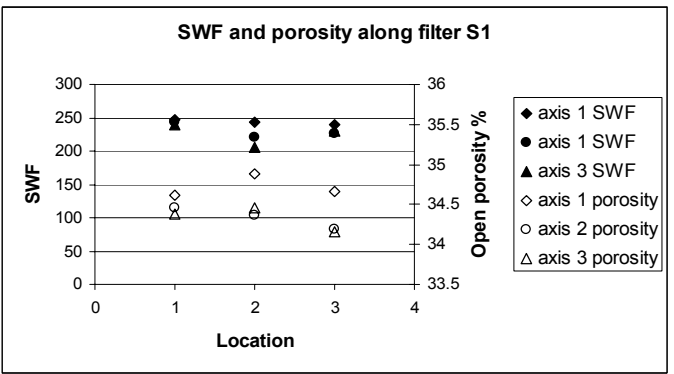

(c)

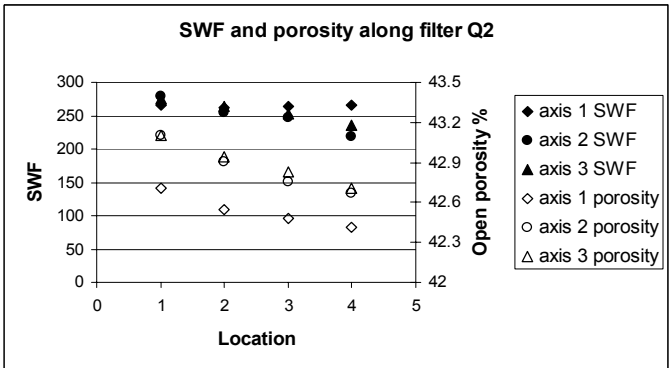

(b)

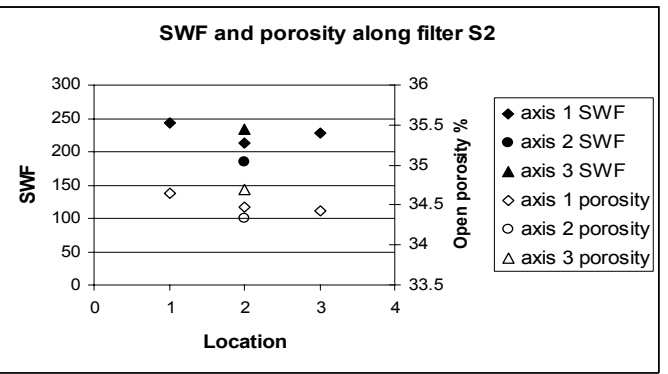

(d)

Fig. 3. Local SWF values and open porosity along the filter samples: (a) Q1, (b) Q2, (c) S1, and (d) S2.

\section{DISCUSSION}

From Figs. 2 and 3 it is evident that the filters are not uniform in terms of porosity. The changes in porosity in Fig. 3 are not large but are clearly more that the standard deviation of the porosity data. Archimedes method gives an averaged value of the local variation seen within the specimen SEM images (Fig. 2). Aduda and Rawlings ${ }^{8}$ reported that the decrease in normalized ring-down count with increasing porosity of sintered glass was due to attenuation by scattering from the pores. Further, both porosity and pore size and shape were found to affect the ring-down counts. In Fig. 3, the SWF along the filter samples is plotted against the averages of open porosity and median strength for each location (SWF values determined from three axes in Fig. 3). The data in Fig. 4 suggest that the variation in SWF along the filter may be influenced by the porosity for filter Q but not significantly for filter S. Further, in material Q an increase in porosity increases the SWF. This contradicts previous results for sintered glass, ${ }^{8}$ but Kathrina and Rawlings ${ }^{9}$ found increasing ring-down count with increasing porosity for sintered $\mathrm{MgO}$.

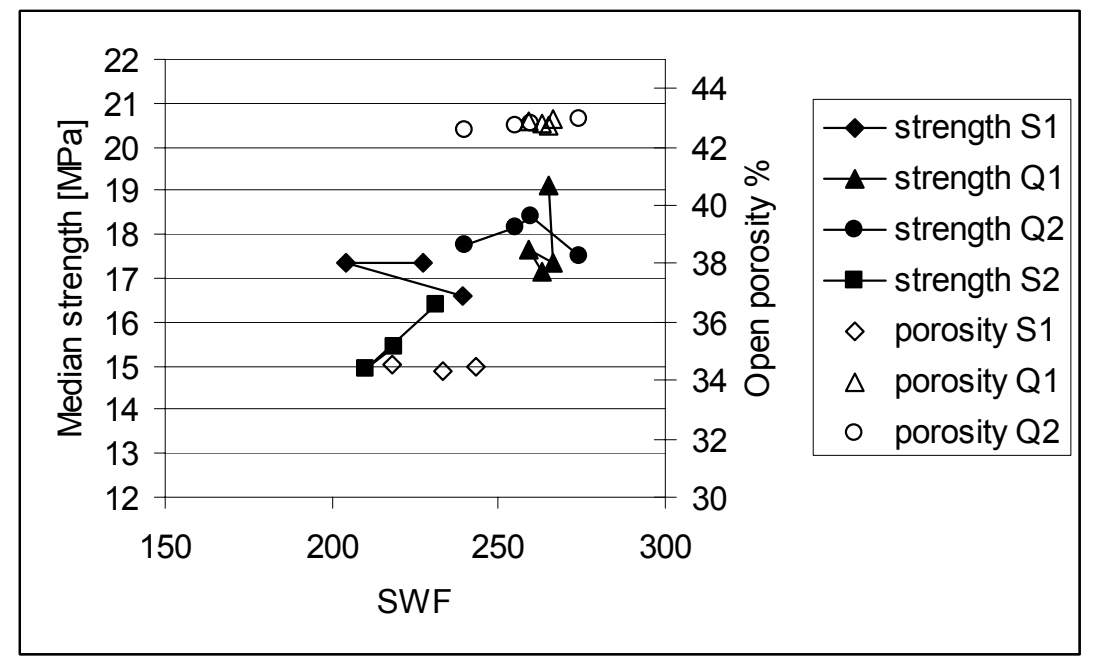

Fig. 4. Median strength of four hoop strength specimens and open porosity associated with with each location as a function of local SWF. (Insufficient data to include porosity 52.) 
It cannot be concluded that porosity would determine the strength of filter Q since the local SWF is not correlated to the median strength associated with each particular location, or that SWF reflects the porosity variation only since the overall SWF does follow the MOR estimate of strength. Scattering may not be the only factor affecting the energy of the wave and, thus, SWF. The influence of porosity variation on the SWF data for sample Q2 can explain the high standard deviation in Table I.

The data for filter S1 suggest that porosity variation is not significant for the SWF. While not enough porosity data are available for filter S2, it is reasonable to assume that porosity has a similar effect to SWF as for filter $\mathrm{S} 1$. The median strength of every four strength specimens associated with each local SWF does not follow SWF for filter S1 but has a strong correlation for filter S2. The variation in strength along filter S2 explains the large standard deviation in the overall SWF (Table I). The strong correlation between local SWF and local strength for S2 suggests that both factors are dominated by the same microstructural feature. That feature could be microcracking since no significant difference in phase content or composition exists for filters S1 and S2.

\section{SUMMARY}

In this work the filter samples from alumina/mullite and clay-bonded $\mathrm{SiC}$ hot gas filters exposed to hightemperature air and air with water vapor were examined with the AU nondestructive evaluation method. The SWF, a ring-down count of amplitudes exceeding a preset threshold value of the detected ultrasound pulse, was determined for several locations along the samples. The variation in the local SWF values was studied in terms of strength, porosity and microstructure of the samples. The overall SWF average was found to correlate with the MOR strength, even though the change in MOR strength due to the exposures was very small. Both filter materials were nonuniform in terms of porosity. The results suggest that local variations of porosity in the alumina/mullite filter could explain some of the variation in local SWF values along the filter samples, but porosity does not determine the strength of the material. In clay-bonded $\mathrm{SiC}$ material exposed to hightemperature air with water vapor, the local SWF values followed the change in median strength of the sections.

The acousto-ultrasound method is capable of monitoring the changes in strength of hot gas filters, and some understanding of the microstructure-SWF-strength relation has been gained, but further work is needed to more firmly establish the microstructural features affecting the strength and SWF of hot gas filter materials.

\section{ACKNOWLEDGMENTS}

The authors wish to acknowledge the Finnish Academy, TEKES, the National Technology Agency, Fortum Power and Heat Oy, and Foster Wheeler Energia Oy for financial support and co-operation in Finland. Work at Argonne National Laboratory was supported by the U.S Department of Energy, Office of Fossil Energy/Advanced Research and Technologies/Materials Program and the Hot Gas Clean-up Program, under contract W-31-109-Eng-38.

\section{REFERENCES}

1. M. A. Alvin, T. E. Lippert, and J. E. Lane, "Assessment of Porous Ceramic Materials for Hot Gas Filtration Applications," Ceramic Bulletin, 70 [9], 1491-1498 (1991).

2. J. E. Oakey and I. R. Fantom, "Hot Gas Cleaning - Materials and Performance," Materials at High Temperatures, 14 [3], 337-345 (1997).

3. P. Pastila, V. Helanti, A.-P. Nikkilä, and T. Mäntylä, "Environmental Effects on Microstructure and Strength of SiC-based Hot Gas Filters," Journal of European Ceramic Society, 21 1261-1268 (2001).

4. W. A. Ellingson, E. R Koehl, J. G. Sun, C. Deemer, H. Lee, and T. Spohnholtz, "Development of Nondestructive Evaluation Methods for Hot Gas Filters," Materials at High Temperatures, 16 [4], 213-218 (1999).

5. W. A. Ellingson, E. R. Koehl, B. Budzynski and J. Carroll, "Development of NDE Technology for Hot Gas Filters," Presented at the $14^{\text {th }}$ Annual Conference on Fossil Energy Materials, Knoxville, TN, April 24-26, 2000 .

6. W. A. Ellingson, E. R. Koehl, D. Sandberg, P. Pastila, A.-P. Nikkilä, and T. A. Mäntylä, "AcoustoUltrasound Nondestructive Characterization of Rigid Ceramic Hot Gas Filters," in Euro Ceramics VII, Key Engineering Materials, Vols. 206-213, pp. 613-616, Trans Tech Publications, Switzerland (2002).

7. D. Gross and C. H. Zhang, "Wave Propagation in Damaged Solids," International Journal of Solids and Structures 29 [14/15], 1763-1779 (1992).

8. B. O. Aduda and R. D. Rawlings, "An Acousto-Ultrasonic Study of the Effect of Porosity on a Sintered Glass System,” Journal of Materials Science, 29 [9], 2297-2303 (1994).

9. T. Kathrina and R. D. Rawlings, "Non-destructive Evaluation of Porous MgO Ceramics Using Acoustic Techniques," Journal of Materials Science, 32 [15], 3951-3959 (1997). 
10. V. Helanti, T. Mäntylä, J. Isaksson, T. Eriksson, and P. Sthåhlberg, "Strength Testing of Tubular Ceramic Gas Filters by Internal Hydraulic Pressure Test," Proceedings of the Second International Conference on Ceramics in Energy Applications," The Institute of Energy, London, pp. 331-337 (1994). 